\title{
ON THE APPLICATIONS OF COALGEBRAS TO GROUP ALGEBRAS
}

\section{ALAN ROSENBERG}

ABSTRACT. This paper examines the coalgebra structure on $k[G]^{\circ}$ and relates it to the group theoretic properties of $G$. In particular it is shown that there is an intimate relation between $k[G]$ being proper and $G$ being residually finite. We use this to derive a series of conditions on the group to guarantee it being residually finite.

It is well known that the dual of a finite dimensional algebra has a canonical coalgebra structure induced on it. This, and the fact that there is a natural isomorphism from $k[G]$ onto $k[G]^{*}$, when $G$ is finite, has been taken advantage of (cf. [7]) in establishing a coalgebra structure on $k[G]$. The comultiplication is

$$
\Delta \sigma=\sum_{\chi \in G} \sigma \chi \otimes \chi^{-1} \quad \forall \sigma \in G
$$

and the counit is

$$
\epsilon\left(\sum n_{\sigma} \sigma\right)=n_{e}
$$

where $e$ is the identity element of $G$. We would like to generalize this to the infinite case.

Unfortunately, the dual of an infinite dimensional algebra is too large to canonically take a coalgebra structure. Therefore, if $A$ is an algebra over $k$ we look at

$$
A^{\circ}=\left\{f \in A^{*} \mid \mathrm{ker} / f \text { contains a cofinite ideal }\right\} .
$$

An ideal $J \subseteq A$ is said to be cofinite if $A / J$ is finite dimensional over $k$. Now there is a canonical way to impose a coalgebra structure on $A^{\circ}$. In fact one may view ()$^{\circ}$ and ()$^{*}$ as adjoint functors between algebras and coalgebras.

Let $i$ be the natural mapping from $k[G]$ into $k[G]^{*}$, given by

$$
i:\{f: G \rightarrow k \mid f \text { has finite support }\} \rightarrow\{f: G \rightarrow k\} \text {. }
$$

Now several questions arise. What does $k[G]^{\circ}$ look like? The following theorem is a step in that direction.

Theorem 1. Let $G$ be an infinite group. Then $i(k[G]) \cap k[G]^{\circ}=(0)$.

Received by the editors May 29, 1974 and, in revised form, July 17, 1974.

AMS (MOS) subject classifications (1970). Primary 16A24, 16A26; Secondary 20E $15,20 \mathrm{E} 25$.

Key words and phrases. Coalgebra, group algebra, proper, residually finite. 
Proof. It can be shown (cf. $\left[6\right.$, p. 115]) that $k[G]^{*}$ is a $k[G]$ module by

$$
(b \rightarrow f)(\sigma)=\sum_{\psi} f(\psi) b\left(\sigma^{-1} \psi\right), \quad b \in k[G], \quad f \in k[G]^{*}, \quad \sigma \in G .
$$

Notice that the sum in (1) is finite. If $*$ is the well-known involution on the group algebra and $f \in k[G]$, then equation (1) says that

$$
b \rightarrow f=f b^{*}
$$

where the right side of (2) is just ordinary multiplication in $k[G]$. It is well known that $f \in k[G]^{*}$ will actually be in $k[G]^{\circ}$ if and only if $k[G] \rightarrow f$ is finite dimensional. By (2), this is equivalent to saying that $f \in i(k[G]) \cap k[G]^{\circ}$ if and only if the right ideal in $k[G]$ generated by $f$ is finite dimensional. But if $G$ is infinite it is easy to see that no right ideal can be finite dimensional. Q.E.D.

Now if $A$ is an algebra then we have a natural mapping, $\rho: A \rightarrow\left(A^{\circ}\right)^{*}$ as algebras. If $\rho$ is one-to-one we say that $A$ is proper. It can be shown that this is equivalent to every nonzero element of $A$ being disjoint from a cofinite ideal. This raises the question: What are necessary and sufficient conditions on $G$ in order that $k[G]$ be proper?

It can be shown, using Krull's intersection theorem and the Hilbert nullstellensatz, that a commutative finitely generated algebra is proper. Since a finitely generated Abelian group is residually finite the following theorem extends this result in the case of the group algebra.

Theorem 2. If $G$ is residually finite then $k[G]$ is proper.

Proof. Let $f \neq 0 \in k[G]$. Consider the set $S=\left\{\sigma \psi^{-1}\right\}$ where $\sigma \neq \psi$ runs through supp $f$. By hypothesis there exists a normal subgroup $N$ of $G$ such that $[G: N]$ is finite and $N$ is disjoint from $S$. Let $\pi$ be the natural projection of $k[G]$ onto $k[G / N]$. Since no two elements of supp $f$ are congruent modulo $N$ we clearly have $f \notin \operatorname{ker} \pi$. Yet $k[G] / \operatorname{ker} \pi \approx k[G / N]$ and so $\operatorname{ker} \pi$ is cofinite. Q.E.D.

Hence $k[G]$ is proper for free groups and finitely generated matrix groups over a commutative ring.

The above theorem possesses a partial converse.

Theorem 3. If $k[G]$ is proper then $G$ is residually finite if either

(i) $k$ is finite, or

(ii) $G$ is torsion and finitely generated.

Proof. Let $\delta$ be any nonidentity element of $G$. We must exhibit a normal subgroup, $N$, which is of finite index in $G$ that does not contain $\delta$. Consider the element $\delta-1$. Since $k[G]$ is proper there exists a cofinite ideal $M$ such that $\delta-1 \notin M$. Consider the map $\rho: G \rightarrow$ Aut $_{k}(k[G] / M)$ defined by $\langle\rho(\iota)$, $f+M\rangle=\imath f+M$ where $\iota \in G$ and $f \in k[G]$. Clearly $\rho$ is a homomorphism to 
the general linear group $\mathrm{Gl}_{n}(k)$ where $n=\operatorname{codim}_{k} M$. Let $N=\operatorname{ker} \rho$. Then $N$ is a normal subgroup of $G$ and it is easy to see that $N=\{\psi \in G \mid \psi-1 \in M\}$. Hence $\delta \notin N$. Now $G / N$ is a subgroup of $\mathrm{Gl}_{n}(k)$. If condition (i) prevails then of course $\mathrm{Gl}_{n}(k)$ and hence also $G / N$ is finite. If case (ii) is true, a theorem of Burnside yields the desired result. (cf. [2]) Q.E.D.

We now proceed to derive sufficient conditions on $G$ for $k[G]$ to be proper.

Theorem 4. If $G$ is a group and its center $Z$ is both finitely generated and of finite index, then $k[G]$ is proper.

Proof. Let $\left\{e=\sigma_{0}, \sigma_{1}, \ldots, \sigma_{n}\right\}$ be a set of coset representatives for $Z$ in $G$, where $e$ is the group identity. Since $k[G]$ is a free $k[Z]$ module with $e, \sigma_{1}, \ldots, \sigma_{n}$ as a basis, if $f \in k[G]$ is nonzero we may write $f=\sum_{i=0}^{n} h_{i} \sigma_{i}$, $h_{i} \in k[Z]$, and not all the $h_{i}$ are zero. If $h_{i} \neq 0$, then since $k[Z]$ is proper, there is a cofinite ideal $J_{i}$ of $k[Z]$ such that $b_{i} \notin J_{i}$. Let $M=\bigcap J_{i}$. Then $M$ is a cofinite ideal of $k[Z]$ and no nonzero $b_{i} \in M$. Consider the set $L=$ $\left\{\Sigma g_{i} \sigma_{i} \mid g_{i} \in M\right\}$. Then since the $g_{i}$ are central we see that $L$ is an ideal. Furthermore, by freeness, $\operatorname{dim}_{k} k[G] / L=\left(\operatorname{dim}_{k} k[G] / M\right) \cdot([G: Z])$. Finally, it is clear that $f \notin$ L. Q.E.D.

Theorem 5. If $G$ is a finitely generated torsion-free nilpotent group then $k[G]$ is proper.

Proof. By a result of Jennings (cf. [3]), the conditions imposed upon $G$ in the hypothesis imply that $\bigcap_{n} \Delta^{n}=(0)$ where $\Delta$ is the fundamental ideal. Hence our result will follow if we can show that each $\Delta^{n}$ is cofinite. We proceed by induction. $\Delta$ is of codimension one. Now assume that $\Delta^{n-1}$ is cofinite. Note that $\Delta / \Delta^{n}$ is a finitely generated $k[G] / \Delta^{n-1}$ module. Since $k[G] / \Delta^{n-1}$ is itself finite dimensional over $k$ it now follows that so is $\Delta / \Delta^{n}$. But we can now conclude that $\Delta^{n}$ is cofinite.

Theorem 6. If $G$ is a finitely generated group and has an Abelian subgroup $H$, of finite index, then $k[G]$ is proper if either

(i) characteristic of $k$ is zero, or

(ii) characteristic of $k$ is $p$ and $G$ contains no elements of order $p$.

Proof. In [5] it is shown that under the assumptions of the hypothesis the simplicial radical of $k[G]$ is trivial. Hence any nonzero element of $k[G]$ is not included in some maximal ideal. But it is well known that the first two conditions on $G$ imply that $k[G]$ is a finitely generated polynomial identity algebra. Theorem 3.3(c) of [4] now guarantees that each maximal ideal is cofinite. Q.E.D.

The previous four theorems combine to yield sufficient conditions for a group to be residually finite.

Theorem 7. A group $G$ is residually finite if it possesses any of the following properties: 
(i) The center of $G$ is both finitely generated and of finite index.

(ii) $G$ is finitely generated, torsion free and nilpotent.

(iii) $G$ is finitely generated, torsion and contains an Abelian subgroup of finite index.

(iv) $G$ is finitely generated, has no $p$ torsion elements for some prime $p$, and contains an Abelian subgroup of finite index.

(The second condition is not a new result and was proved by Gruenberg (cf. [1]), although in a different manner.)

\section{REFERENCES}

1. K. W. Gruenberg, Residual properties of infinite soluble groups, Proc. London Math. Soc. (3) 7 (1957), 29-62. MR 19, 386.

2. I. N. Herstein, Noncommutative rings, Carus Math. Monos., no. 15, Math. Assoc. Amer. (distributed by Wiley, New York), 1968. MR $37 \# 2790$.

3. S. A. Jennings, The group ring of a class of infinite nilpotent groups, Canad. J. Math 7 (1955), 169-187. MR 16, 899.

4. Claudio Procesi, Non commutative Jacobson-rings, Ann. Scuola Norm. Sup. Pisa (3) 21 (1967), 281-290. MR 37 \#251.

5. Alan Rosenberg, The simplicial radical of the group algebra (unpublished).

6. Moss Sweedler, Hopf algebras, Math. Lecture Notes Ser., Benjamin, New York, 1969. MR $40 \# 5705$.

7. E. J. Taft, Reflexivity of algebras and coalgebras, Amer. J. Math. 94 (1972), 1111-1130. MR 46 \#9095.

DEPARTMENT OF MATHEMATICS, WESLEYAN UNIVERSITY, MIDDLETOWN, CONNEC. TICUT 06457 